\title{
De Sitter Entropy as Holographic Entanglement Entropy
}

\author{
Nikolaos Tetradis ${ }^{1 \star}$ \\ 1 Department of Physics, National and Kapodistrian University of Athens, \\ University Campus, Zographou 157 84, Greece \\ $\star$ [ntetrad@phys.uoa.gr] \\ 4th International Conference on Holography, \\ String Theory and Discrete Approach \\ Hanoi, Vietnam, 2020 \\ doi:10.21468/SciPostPhysProc.4
}

\begin{abstract}
We review the results of refs. [1,2], in which the entanglement entropy in spaces with horizons, such as Rindler or de Sitter space, is computed using holography. This is achieved through an appropriate slicing of anti-de Sitter space and the implementation of a UV cutoff. When the entangling surface coincides with the horizon of the boundary metric, the entanglement entropy can be identified with the standard gravitational entropy of the space. For this to hold, the effective Newton's constant must be defined appropriately by absorbing the UV cutoff. Conversely, the UV cutoff can be expressed in terms of the effective Planck mass and the number of degrees of freedom of the dual theory. For de Sitter space, the entropy is equal to the Wald entropy for an effective action that includes the higher-curvature terms associated with the conformal anomaly. The entanglement entropy takes the expected form of the de Sitter entropy, including logarithmic corrections.
\end{abstract}

Copyright N.Tetradis.

This work is licensed under the Creative Commons

Attribution 4.0 International License.

Published by the SciPost Foundation.
Received ??-??-20??

Accepted ??-??-20??

Published ??-??-20??

doi:10.21468/SciPostPhysProc.4.??

\section{Contents}

The fact that the divergent part of the entanglement entropy scales with the area of the entangling surface [3] suggests a connection with the gravitational entropy of spaces containing horizons. It seems reasonable that the entropies should become equal when the entangling surface is identified with a horizon. We address this problem in the context of the AdS/CFT correspondence through use of appropriate coordinates that set the boundary metric in Rindler or static de Sitter form. According to the Ryu-Takayanagi proposal [4], the entanglement entropy of a part of the AdS boundary within an entangling surface $\mathcal{A}$ is proportional to the area of a minimal surface $\gamma_{A}$ anchored on $\mathcal{A}$ and extending into the bulk. 

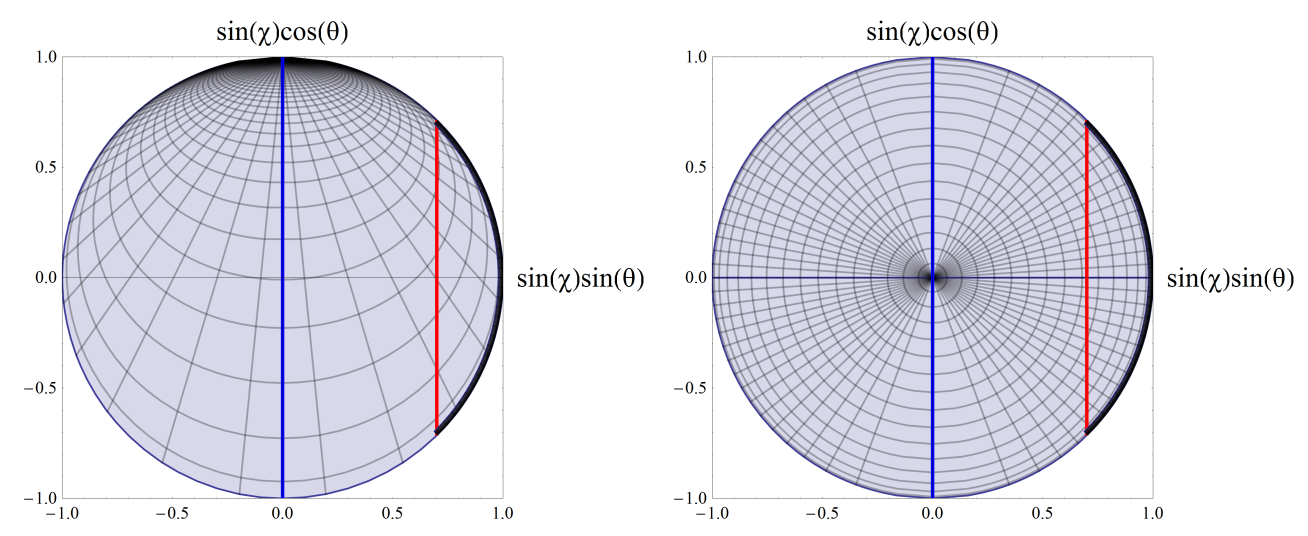

Figure 1: Constant-time slice of $\mathrm{AdS}_{3}$ for a Rindler boundary with $a=1$ (left) and a static de Sitter boundary with $H=1$ (right).

We consider the standard parameterization of $(d+2)$-dimensional AdS space with global coordinates, as well as parametrizations through Fefferman-Graham coordinates, with the boundary located at the value $z=0$ of the bulk coordinate. As a first case we consider a metric with a Rindler boundary:

$$
d s_{d+2}^{2}=\frac{R^{2}}{z^{2}}\left[d z^{2}-a^{2} y^{2} d \eta^{2}+d y^{2}+d \vec{x}_{d-1}\right],
$$

where $a$ is a constant parameter. The timelike coordinate $\eta$ takes values $-\infty<\eta<\infty$. The range $0<y<\infty$ of the spacelike coordinate $y$ covers the right (R) Rindler wedge, while the range $-\infty<y<0$ covers the left (L) wedge.

In the left plot of fig. 1 we depict how the slice of the $\mathrm{AdS}_{3}$ cylinder with $\eta=0$ is covered by the coordinates $y$ and $z$ for $a=1$. The two axes correspond to global coordinates. The circumference is the $\mathrm{AdS}_{3}$ boundary with $z=0$, which is parameterized by the coordinate $y$. The Rindler horizon at $y=0$ corresponds to the point $(0,-1)$ in fig. 1 . Positive values of $y$ cover the right semicircle ( $\mathrm{R}$ wedge), and negative values the left semicircle (L wedge). The point $(0,1)$ is approached in the limits $y \rightarrow \pm \infty$ from right or left. The $\mathrm{AdS}_{3}$ interior is covered by lines of constant $y$ and variable positive $z$. All these lines converge to the point $(0,1)$ for $z \rightarrow \infty$. We expect to have entanglement between the $\mathrm{R}$ and $\mathrm{L}$ wedges. The corresponding entanglement entropy can obtained through holography by computing the area of the minimal surface $\gamma_{A}$ of ref. [4]. This is depicted by the blue line in this case, which acts as a bulk horizon. The Rindler horizon can be viewed as the holographic image of the bulk horizon.

Let us consider a strip with width $l$ in the $y$-direction and very large extent in the remaining spacelike directions. The minimal surface extends into the bulk up to $z_{*}=\Gamma\left(\frac{1}{2 d}\right) /\left(2 \sqrt{\pi} \Gamma\left(\frac{d+1}{2 d}\right)\right) l$. In global coordinates this surface corresponds to a straight line through the bulk, as depicted by the red line in fig. 1 . The entanglement entropy can be computed as

$$
S_{A}=\frac{2 R\left(R^{d-1} L^{d-1}\right)}{4 G_{d+2}}\left(\frac{1}{(d-1) \epsilon^{d-1}}+\frac{\sqrt{\pi}}{2 d} \frac{\Gamma\left(\frac{1-d}{2 d}\right)}{\Gamma\left(\frac{1}{2 d}\right)} \frac{1}{z_{*}^{d-1}}\right) .
$$

A cutoff $\epsilon$ has been imposed on $z$ as the surface approaches the boundary. For $d=1$, one must substitute $1 /\left((d-1) \epsilon^{d-1}\right)$ with $\log (1 / \epsilon)$. Here $L$ is the large length of the directions perpendicular to the strip, so that $R^{d-1} L^{d-1}$ is the corresponding volume.

We are interested in the limit in which the width $l$ of the strip covers the whole R wedge. In this case the entanglement occurs between the R and L wedges. For $l \rightarrow \infty$ we have $z_{*} \rightarrow \infty$ 
and the second term in the parenthesis in eq. (2) vanishes. In order to assign a physical meaning to the first term, we can define the effective Newton's constant for the boundary theory as in [5]:

$$
G_{d+1}=(d-1) \epsilon^{d-1} \frac{G_{d+2}}{R},
$$

with $(d-1) \epsilon^{d-1}$ replaced by $1 / \log (1 / \epsilon)$ for $d=1$. This definition can be justified in the context of the Randall-Sundrum (RS) model [6], which employs only the part of the AdS space with $z>\epsilon$. The effective low-energy theory includes dynamical gravity with a Newton's constant given by eq. (3). In the limit $\epsilon \rightarrow 0$, the constant vanishes and gravity becomes non-dynamical. This demonstrates the difficulty in computing the gravitational entropy in the context of the AdS/CFT coorespondence. The resolution we suggest is to keep the cutoff nonzero and absorb it in the definition of the effective Newton's constant. Trading $\epsilon$ for $G_{d+1}$ in the expression for the entropy results in a meaningful expression.

Substituting eq. (3) in eq. (2) gives an entanglement entropy which is bigger by a factor of 2 than the known gravitational entropy [7]. The reason can be traced to the way the limit is taken in order to cover the whole $\mathrm{R}$ wedge. We start from a strip in the $y$-direction extending between two points $y_{1}$ and $y_{2}$, and then take the limits $y_{1} \rightarrow 0$ and $y_{2} \rightarrow \infty$. The first limit leads to the location of the Rindler horizon. However, any finite value of $y_{2}$ excludes an infinite domain corresponding to $y>y_{2}$. As a result, the strip is entangled not only with the (infinite) L wedge, but also with the (infinite) domain $y>y_{2}$. The two contributions are expected to be equal because the space is essentially flat. Obtaining the entropy corresponding to the entanglement with the L wedge only can be obtained by dividing the result with a factor of 2 . The final result for the Rindler entropy is

$$
S_{R}=\frac{R^{d-1} L^{d-1}}{4 G_{d+1}},
$$

in agreement with [7]. It is also illuminating to observe that the bulk horizon depicted as a blue line in fig. 1 approaches the boundary at two points. The point $(0,-1)$ is the true Rindler horizon. However, the point $(0,1)$ does not belong to the boundary Rindler space, but corresponds only to the limits $y \rightarrow \pm \infty$. The contribution to the area of the entangling surface from its vicinity should not be taken into account, thus justifying the division by 2 .

The second case we consider is that of a boundary static de Sitter (dS) space:

$$
d s_{d+2}^{2}=\frac{R^{2}}{z^{2}}\left[d z^{2}+\left(1-\frac{1}{4} H^{2} z^{2}\right)^{2}\left(-\left(1-H^{2} \rho^{2}\right) d t^{2}+\frac{d \rho^{2}}{1-H^{2} \rho^{2}}+\rho^{2} d \Omega_{d-1}^{2}\right)\right] .
$$

For $d>1$, the range $0 \leq \rho \leq 1 / H$ covers one static patch. There are two such patches in the global geometry, which start from the the "North" or "South pole" at $\rho=0$ and are joined at the surface with $\rho=1 / H$. For $d=1, \rho$ can also take negative value and each static patch is covered by $-1 / H \leq \rho \leq 1 / H$. In the right plot of fig. 1 we depict how the slice of the $\mathrm{AdS}_{3}$ cylinder with $t=0$ is covered by the coordinates $\rho$ and $z$ for $H=1$. The circumference is again the $\mathrm{AdS}_{3}$ boundary with $z=0$, which is parameterized by the coordinate $\rho$. There are two horizons: one at $\rho=-1$, corresponding to the point $(0,-1)$, and one at $\rho=1$, corresponding to the point $(0,1)$ on the boundary. The $\mathrm{AdS}_{3}$ interior is covered by lines of constant $\rho$ and variable positive $z$. All these lines converge to the point $(0,0)$ at the center for $z \rightarrow \infty$. In the context of the global geometry, we expect to have entanglement between the two static patches. The corresponding entanglement entropy can be obtained through holography by computing the area of the minimal surface $\gamma_{A}$ of ref. [4], depicted by the blue line. This line acts as bulk horizon. The difference with the Rindler case we discussed before is that the endpoints of the minimal surface are points of the boundary dS space, they are actually the 
horizons. This means that there is no need to divide by a factor of 2 in this case. For $d>1$ the $d$-dimensional minimal surface $\gamma_{A}$ ends on an $(d-1)$-dimensional sphere that separates the two hemispheres of the slice of $\mathrm{dS}_{d+1}$ with $t=0$.

The isometries of dS space indicate that the entangling surface is spherical in this case. The minimal surface $\gamma_{A}$ in the bulk can be determined by minimizing the integral

$$
\operatorname{Area}\left(\gamma_{A}\right)=R^{d} S^{d-1} \int d \sigma \frac{\sin ^{d-1}(\sigma)}{\sinh ^{d}(w)} \sqrt{1+\left(\frac{d w(\sigma)}{d \sigma}\right)^{2}},
$$

where we have defined the parameters $\sigma=\sin ^{-1}(H \rho), w=2 \tanh ^{-1}(H z / 2)$, and denoted the volume of the $(d-1)$-dimensional unit sphere as $S^{d-1}$. The above expression is minimized by the function [2]

$$
w(\sigma)=\cosh ^{-1}\left(\frac{\cos (\sigma)}{\cos \left(\sigma_{0}\right)}\right) .
$$

For $\sigma_{0} \rightarrow 0$ the known expression $w(\sigma)=\sqrt{\sigma_{0}^{2}-\sigma^{2}}$ [4] for $H=0$ is reproduced. For $\sigma_{0} \rightarrow \pi / 2$ the boundary is approached at the location of the horizon with $d w / d \sigma \rightarrow-\infty$.

The integral (6) is dominated by the region near the boundary. Introducing a cutoff at $z=\epsilon$ results in a leading contribution

$$
\operatorname{Area}\left(\gamma_{A}\right)=R^{d} S^{d-1} I(\epsilon)=R^{d} S^{d-1} \int_{H \epsilon} \frac{d w}{\sinh ^{d}(w)} .
$$

For $d \neq 1$ the leading divergent part is $I(\epsilon)=1 /\left((d-1) H^{d-1} \epsilon^{d-1}\right)$, while for $d=1$ it is $\log (1 /(H \epsilon))$. Using eq. (3) we obtain the leading contribution to the entropy:

$$
S_{\mathrm{dS}}=\frac{\operatorname{Area}\left(\gamma_{A}\right)}{4 G_{d+2}}=\frac{R^{d} S^{d-1}}{4 G_{d+2}(d-1) H^{d-1} \epsilon^{d-1}}=\frac{S^{d-1}}{4 G_{d+1}}\left(\frac{R}{H}\right)^{d-1}=\frac{A_{H}}{4 G_{d+1}},
$$

with $A_{H}$ the area of the horizon. This result reproduces the gravitational entropy of [8]. It is valid for $d=1$ as well, with $1 /\left((d-1) \epsilon^{d-1}\right)$ replaced by $\log (1 / \epsilon)$ and $S^{0}=2$, because the horizons of the global $\mathrm{dS}_{2}$ geometry are 2 points [5].

The integral $I(\epsilon)$ also contains subleading divergences. There is a subleading logarithmic divergence for $d=3$, no singular subleading terms for $d=2$, while the only divergence for $d=1$ is the leading logarithmic term already included in eq. (9). For $d>3$ we have subleading power-law divergences for odd $d+1$, plus a logarithmic one for even $d+1$. We focus on four dimensions, in which the dS entropy takes the form

$$
S_{\mathrm{dS}}=\frac{A_{H}}{4 G_{4}}\left(1+H^{2} \epsilon^{2} \log H \epsilon\right) \text {. }
$$

The logarithmic dependence on the cutoff hints at a connection with the conformal anomaly of the dual theory, which results from higher curvature terms in the effective theory. The effective action can be deduced from known results for the on-shell action in holographic renormalization [9]. In our approach the divergences are not removed through the introduction of counterterms, but are absorbed in the effective couplings. This means that the relevant quantity for our purposes is the regulated form of the effective action. Using the results of [9], we obtain the leading terms [2]

$$
S=\frac{R^{3}}{16 \pi G_{5}} \int d^{4} x \sqrt{-\gamma}\left[\frac{6}{\epsilon^{4}}+\frac{1}{2 \epsilon^{2}} \mathcal{R}-\frac{1}{4} \log \epsilon\left(\mathcal{R}_{i j} \mathcal{R}^{i j}-\frac{1}{3} \mathcal{R}^{2}\right)\right] .
$$

The first term corresponds to a cosmological constant. In the RS model [6] this is balanced by the surface tension of the brane at $z=\epsilon$. The second term is the standard Einstein term if the 
effective Newton's constant $G_{4}$ is defined as in eq. (3) with $d=3$. The third term is responsible for the holographic conformal anomaly. The action (11) supports a dS solution. In order to take into account the presence of the higher-curvature terms in eq. (11) one must compute the Wald entropy [10]. The result is in agreement with the singular part of the correction provided by the holographic calculation (10) [2].

For the $\mathcal{N}=4$ supersymmetric $S U(N)$ gauge theory in the large- $N$ limit, the effective action can be computed as [11]

$$
S=-\frac{\beta}{16 \pi^{2}} \Gamma\left(2-\frac{d+1}{2}\right) \int d^{4} x \sqrt{-\gamma}\left(\mathcal{R}_{i j} \mathcal{R}^{i j}-\frac{1}{3} \mathcal{R}^{2}\right),
$$

with $\beta=-N^{2} / 4$. The divergence of $\Gamma(2-(d+1) / 2)$ in dimensional regularization in the limit $d+1 \rightarrow 4$ corresponds to a $\log \left(1 / \epsilon^{2}\right)$ divergence in our cutoff regularization. A comparison of the above expression with eq. (11) reproduces the standard AdS/CFT relation $G_{5}=\pi R^{3} /\left(2 N^{2}\right)$. The dimensionful UV momentum cutoff for $d=3$ can be expressed as $\left(\epsilon_{N} R\right)^{-2}=2 G_{5} /\left(R^{3} G_{4}\right)=8 \pi^{2} m_{\mathrm{Pl}}^{2} / N^{2}$, with $m_{\mathrm{Pl}}^{2}=1 /\left(8 \pi G_{4}\right)$. Now eq. (10) for $d=3$ can be cast in the form

$$
S_{\mathrm{dS}}=\frac{A_{H}}{4 G_{4}}+N^{2} \log \left(H \epsilon_{N}\right)=\frac{A_{H}}{4 G_{4}}+N^{2} \log \left(\frac{N}{\sqrt{8} \pi} \frac{H / R}{m_{\mathrm{Pl}}}\right),
$$

where $H / R$ is the physical Hubble scale. This expression is completely analogous to the blackhole result [12], with the horizon size parameter measured in units of the UV cutoff. It is also in agreement with the calculation of the logarithmic part of the holographic entanglement entropy in [13].

The calculation of the entropy associated with nontrivial gravitational backgrounds through holography faces two difficulties:

- The boundary metric in the context of AdS/CFT is not dynamical, a feature that is equivalent to $m_{\mathrm{Pl}} \rightarrow \infty$.

- The entanglement entropy has a strong dependence on the UV cutoff of the theory, which makes its identification with the gravitational entropy problematic.

We showed that these difficulties can be resolved if the UV cutoff dependence is absorbed in the definition of $m_{\mathrm{Pl}}$. The conceptual framework is provided by the Randall-Sundrum model [6], or, alternatively, by the regulated form of the effective action in holographic renormalization [9]. Our derivation of the dS entropy is consistent with the expectation that the entropy associated with gravitational horizons can be understood as entanglement entropy if Newton's constant is induced by quantum fluctuations of matter fields [14]. In the context of the AdS/CFT correspondence the bulk degrees of freedom correspond to the matter fields of the dual theory. The boundary Einstein action arises through the integration of these bulk degrees of freedom up to the UV cutoff.

Our approach is in contrast with the usual interpretation of the leading contribution to the entanglement entropy as an unphysical UV-dependent quantity of little interest. We have reached the opposite conclusion: The leading contribution to the entropy has a universal form that depends only on the horizon area because the same degrees of freedom contribute to the entropy and Newton's constant. Also, the detailed nature of the UV cutoff does not affect the leading contribution. The particular features of the underlying theory, such as the number of degrees of freedom become apparent at the level of the subleading corrections to the entropy: the coefficient of the logarithmic correction is determined by the central charge of the theory. 


\section{Acknowledgments}

This research work was supported by the Hellenic Foundation for Research and Innovation (H.F.R.I.) under the First Call for H.F.R.I. Research Projects to support Faculty members and Researchers (Project Number: 824).

\section{References}

[1] D. Giataganas and N. Tetradis, Phys. Lett. B 796 (2019), 88-92 [arXiv:1904.13119 [hepth]].

[2] N. Tetradis, Phys. Lett. B 807 (2020), 135552 [arXiv:1910.10587 [hep-th]].

[3] L. Bombelli, R. K. Koul, J. Lee and R. D. Sorkin, Phys. Rev. D 34 (1986) 373; M. Srednicki, Phys. Rev. Lett. 71 (1993) 666 [hep-th/9303048].

[4] S. Ryu and T. Takayanagi, Phys. Rev. Lett. 96 (2006) 181602 [hep-th/0603001]; T. Nishioka, S. Ryu and T. Takayanagi, J. Phys. A 42 (2009) 504008 [arXiv:0905.0932 [hep-th]]; S. Ryu and T. Takayanagi, JHEP 0608 (2006) 045 [hep-th/0605073].

[5] S. Hawking, J. M. Maldacena and A. Strominger, JHEP 0105 (2001) 001 [hepth/0002145].

[6] L. Randall and R. Sundrum, Phys. Rev. Lett. 83 (1999) 3370 [hep-ph/9905221]; Phys. Rev. Lett. 83 (1999) 4690 [hep-th/9906064].

[7] R. Laflamme, Phys. Lett. B 196 (1987) 449.

[8] G. W. Gibbons and S. W. Hawking, Phys. Rev. D 15 (1977) 2738.

[9] S. de Haro, S. N. Solodukhin and K. Skenderis, Commun. Math. Phys. 217 (2001) 595 [hep-th/0002230]; K. Skenderis, Class. Quant. Grav. 19 (2002) 5849 [hep-th/0209067]; I. Papadimitriou and K. Skenderis, IRMA Lect. Math. Theor. Phys. 8 (2005) 73 [hepth/0404176].

[10] R. M. Wald, Phys. Rev. D 48 (1993) no.8, R3427 [gr-qc/9307038]; V. Iyer and R. M. Wald, Phys. Rev. D 50 (1994) 846 [gr-qc/9403028]; T. Jacobson, G. Kang and R. C. Myers, Phys. Rev. D 49 (1994) 6587 [gr-qc/9312023].

[11] N. D. Birrell and P. C. W. Davies, Quantum fields in curved space, Cambridge Monographs on Mathematical Physics, Cambridge University Press, 1982.

[12] A. Sen, JHEP 1304 (2013) 156 [arXiv:1205.0971 [hep-th]].

[13] H. Casini, M. Huerta and R. C. Myers, JHEP 1105 (2011) 036 [arXiv:1102.0440 [hepth]].

[14] L. Susskind and J. Uglum, Phys. Rev. D 50 (1994) 2700 [hep-th/9401070]; T. Jacobson, gr-qc/9404039. 